\title{
Wada Dessins associated with Finite Projective Spaces and Frobenius Compatibility
}

\author{
Cristina Sarti \\ Mathematisches Seminar, Goethe Universität \\ Postfach 111932, D-60054 Frankfurt a. M., Germany
}

Received 20 October 2009, accepted 16 July 2011, published online 19 August 2011

\begin{abstract}
Dessins d'enfants (hypermaps) are useful to describe algebraic properties of the Riemann surfaces they are embedded in. In general, it is not easy to describe algebraic properties of the surface of the embedding starting from the combinatorial properties of an embedded dessin. However, this task becomes easier if the dessin has a large automorphism group.

In this paper we consider a special type of dessins, so-called Wada dessins. Their underlying graph illustrates the incidence structure of finite projective spaces $\mathbb{P}^{m}\left(\mathbb{F}_{n}\right)$. Usually, the automorphism group of these dessins is a cyclic Singer group $\Sigma_{\ell}$ permuting transitively the vertices. However, in some cases, a second group of automorphisms $\Phi_{f}$ exists. It is a cyclic group generated by the Frobenius automorphism. We show under what conditions $\Phi_{f}$ is a group of automorphisms acting freely on the edges of the considered dessins.

Keywords: Dessins d'enfants, Wada dessins, bipartite graphs, graph embeddings, difference sets, finite geometries, Riemann surfaces, Frobenius automorphism, Singer groups.
\end{abstract}

Math. Subj. Class.: 05C10, 30F10, 05B10, 05B25, 51E20, $51 D 20$

\section{Introducing dessins d'enfants}

The term dessins d'enfants was first used by Grothendieck (1984) to refer to objects which are very simple but important to describe Riemann surfaces. Dessins d'enfants $\mathcal{D}$ can be defined as hypermaps on compact orientable surfaces. A hypermap in its Walsh representation [15] is a bipartite graph drawn without crossings on a surface $X$ and cutting the surface into simply connected cells (faces).

For a vertex of the graph we call valency the number of incident edges. For a cell, the valency is the number of edges on its boundary. This number is always even for hypermaps.

E-mail address: sarti@math.uni-frankfurt.de (Cristina Sarti) 
Edges bordering a cell from both sides have to be counted twice (see [14] and [11]). A characteristic property of a dessin is its signature $(p, q, r)$, where

1. $p$ is the least common multiple of all valencies of the white vertices,

2. $q$ is the least common multiple of all valencies of the black vertices,

3. $2 r$ is the least common multiple of all face valencies.

A dessin is called uniform if all white vertices have the same valency $p$, all black vertices have the same valency $q$ and all cells have the same valency $2 r$. Riemann surfaces with embedded dessins are algebraic curves of special type. According to Belyı̌'s theorem (see [2]) a surface $X$ admits a model over the field $\overline{\mathbb{Q}}$ if and only if there exists a meromorphic function $\beta$

$$
\beta: X \rightarrow \mathbb{P}^{1}(\mathbb{C})
$$

ramified above at most three points. Without loss of generality, these can be identified with $\{0,1, \infty\}$. The preimages of 0 and 1 are, respectively, the set of white and black vertices of the bipartite graph on $X$. The preimages of $\infty$ correspond to points within the faces. Each face contains exactly one point which is commonly called face center (see e.g. [11]). This means that we have a dessin on a surface if and only if we may describe it with equations whose coefficients are in the field $\overline{\mathbb{Q}}$.

In general, it is not easy to relate the combinatorial properties of a dessin to algebraic properties of the surface, such as defining equations or the moduli field, but the task becomes easier if the embedded dessin has a large automorphism group. In the best case, this group acts transitively on the edges of the dessin (for recent results see, for instance, [10]) and we say that the dessin is not only uniform but even regular.

In this paper, we consider dessins whose underlying graph describes the incidence structure of points and hyperplanes of projective spaces $\mathbb{P}^{m}\left(\mathbb{F}_{n}\right)$ (see Sections 2 and 2.1). The possibility to construct this kind of dessins was first studied by Streit and Wolfart [14] for projective planes $\mathbb{P}^{2}\left(\mathbb{F}_{n}\right)$. Starting from some of their results, we examine here special kinds of uniform dessins called Wada dessins (see Section 2.2). The cells of these dessins have all the same valency $2 \ell$ and there always exists a cyclic group $\Sigma_{\ell}$ transitively permuting the edges of type $\circ-\bullet$ and of type $\bullet-\circ$ on their boundaries (see [14] and [12]). We are interested in determining when the full automorphism group contains other groups of automorphisms beside $\Sigma_{\ell}$.

We consider the Frobenius automorphism acting on points and on hyperplanes of projective spaces $\mathbb{P}^{m}\left(\mathbb{F}_{n}\right)$ (see Section 3) and we establish under what conditions it induces an automorphism of the associated Wada dessins (see Sections 3.1 and 3.2). In general, it is not easy to predict the necessary restrictions on the parameters $m$ and $n$ such that these conditions are satisfied. In Section 4 we prove that if $n$ and $m+1$ are primes the problem can be solved.

Since in the literature automorphisms of dessins are defined in slightly different ways, we point out that here we consider only orientation-preserving automorphisms.

\section{Finite projective spaces}

Let $\mathbb{F}_{n}^{m+1}$ be the vector space over the finite field $\mathbb{F}_{n}, n=p^{e}$ a prime power. We define the finite projective space $\mathbb{P}^{m}\left(\mathbb{F}_{n}\right)$ as the vector space $\mathbb{F}_{n}^{m+1} \backslash\{0\}$ factorized by the 
multiplicative group $\mathbb{F}_{n}^{*}$ of order $n-1$ :

$$
\mathbb{P}^{m}\left(\mathbb{F}_{n}\right):=\left(\mathbb{F}_{n}^{m+1} \backslash\{0\}\right) / \mathbb{F}_{n}^{*}
$$

The integer $n$ is called order of $\mathbb{P}^{m}\left(\mathbb{F}_{n}\right)$ ([3], [5]).

The number of points of $\mathbb{P}^{m}\left(\mathbb{F}_{n}\right)$ is given by

$$
\ell:=\left|\mathbb{P}^{m}\left(\mathbb{F}_{n}\right)\right|=\frac{\left|\mathbb{F}_{n}^{m+1} \backslash\{0\}\right|}{\left|\mathbb{F}_{n}^{*}\right|}=\frac{n^{m+1}-1}{n-1} .
$$

By duality, the integer $\ell$ is also the number of hyperplanes of $\mathbb{P}^{m}\left(\mathbb{F}_{n}\right)$.

Hyperplanes are subspaces $\mathbb{U}^{m-1}\left(\mathbb{F}_{n}\right)$ of dimension $m-1$ in $\mathbb{P}^{m}\left(\mathbb{F}_{n}\right)$, thus they correspond to subspaces of dimension $m$ in $\mathbb{F}_{n}^{m+1}$. Similarly to equation (2.2), we compute the number of points on each of them as:

$$
q:=\left|\mathbb{U}^{m-1}\left(\mathbb{F}_{n}\right)\right|=\frac{\left|\mathbb{F}_{n}^{m} \backslash\{0\}\right|}{\left|\mathbb{F}_{n}^{*}\right|}=\frac{n^{m}-1}{n-1} .
$$

By duality, the integer $q$ is also the number of hyperplanes through each point.

The finite field $\mathbb{F}_{n^{m+1}}$ may be considered as a vector space over the field $\mathbb{F}_{n}$. A well known fact is that the multiplicative group of a finite field is cyclic. Therefore the $m$ dimensional projective space over $\mathbb{F}_{n}$ may be identified with the quotient

$$
\mathbb{P}^{m}\left(\mathbb{F}_{n}\right) \cong \mathbb{F}_{n^{m+1}}^{*} / \mathbb{F}_{n}^{*},
$$

which, being considered as a group, is also cyclic of order $\ell$. Let $g$ be a generator of this group.

Due to the identification above we make correspond points $P_{b}$ and, by duality, hyperplanes $h_{w}$ of $\mathbb{P}^{m}\left(\mathbb{F}_{n}\right)$ to powers of the generator $g$ :

$$
g^{b} \leftrightarrow P_{b}, \quad g^{w} \leftrightarrow h_{w}, \quad b, w \in \mathbb{Z} / \ell \mathbb{Z} .
$$

In this way, we obtain a numbering of points and of hyperplanes by the integers $b, w \in$ $\{0, \ldots, \ell-1\}$. Point and hyperplane numberings are closely related to each other and are not arbitrary as we will explain more in detail in Section 2.1.

Among the projective linear transformations of $\mathbb{P}^{m}\left(\mathbb{F}_{n}\right)$ we find cyclic projectivities permuting in a single cycle, i.e. transitively, the set of points. By duality they also transitively permutes the set of hyperplanes. These projectivities generate so-called Singer groups (see e.g. [7]). Due to (2.4), it is easy to prove that the group $\Sigma_{\ell} \cong \mathbb{F}_{n^{m+1}}^{*} / \mathbb{F}_{n}^{*}$-with $\ell$ defined as in (2.2) - is cyclic and it acts transitively permuting the set of points and the set of hyperplanes of $\mathbb{P}^{m}\left(\mathbb{F}_{n}\right)$. Hence, we formulate the following

Proposition-Definition 2.1. Every $\mathbb{P}^{m}\left(\mathbb{F}_{n}\right)$ admits a point (hyperplane) transitive cyclic group of automorphisms. This group is a Singer group.

This proposition was first proved by Singer ([13]) for projective planes $\mathbb{P}^{2}\left(\mathbb{F}_{n}\right)$. Now it is widely known ([1], [5], [7]) that it also holds for spaces of higher dimension. For an exhaustive proof see [9, Chapter II, Section 7]. The existence of a Singer group cyclically permuting points and hyperplanes justifies the fact that projective spaces $\mathbb{P}^{m}\left(\mathbb{F}_{n}\right)$ are commonly called cyclic projective spaces. 
Let $\gamma$ be a generating element of $\Sigma_{\ell}$. Thus, due to (2.5) and to (2.4), the action of each element $\gamma^{a} \in \Sigma_{\ell}, a \in \mathbb{Z} / \ell \mathbb{Z}$, on the points $P_{b}$ and on the hyperplanes $h_{w}$ is naturally expressed by:

$$
\begin{aligned}
\gamma^{a}: P_{b} & \longmapsto P_{b+a}, \\
h_{a} & \longmapsto h_{w+a} .
\end{aligned}
$$

\subsection{Constructing dessins}

In order to construct dessins associated with a projective space $\mathbb{P}^{m}\left(\mathbb{F}_{n}\right)$, we need to construct the corresponding bipartite graph. We first introduce the conventions in Table 1.

\begin{tabular}{|l||c|}
\hline point & black vertex $\bullet$ \\
\hline hyperplane & white vertex $\circ$ \\
\hline incidence & joining edge - \\
\hline
\end{tabular}

Table 1: Conventions

Incidence between a point $P_{b}$ and an hyperplane $h_{w}$ is illustrated by the bipartite graph through a joining edge between a black vertex $b$ and a white vertex $w$. Recall that points $P_{b}$ and hyperplanes $h_{w}$ are numbered with integers $b, w \in\{0, \ldots, \ell-1\}$ given by the exponents of a generator $g$ of $\mathbb{F}_{n^{m+1}}^{*} / \mathbb{F}_{n}^{*}$ (see Section 2, Relation (2.5) ). This numbering is not arbitrary. We consider the $q$ points on a line. Thanks to the existence of the Singer group defined in Proposition 2.1, Singer could show (see [13]) that for projective planes the integers resulting from differences of a line index with the indices of the incident points form a difference set. Difference sets are defined in the following way:

Definition 2.2 ([1]). A $(v, k, \lambda)$-difference set $D=\left\{d_{1}, \ldots, d_{k}\right\}$ is a collection of $k$ residues modulo $v$, such that for any residue $\alpha \neq \equiv \bmod v$ the congruence

$$
d_{i}-d_{j} \equiv \alpha \quad \bmod v
$$

has exactly $\lambda$ solution pairs $\left(d_{i}, d_{j}\right)$ with $d_{i}$ and $d_{j}$ in $D$.

In particular, sets $(D+s) \bmod v$ with $s \in \mathbb{Z} / v \mathbb{Z}$ are also difference sets and we call them shifts of $D$. If $\widehat{D} \equiv(t \cdot D+s) \bmod v$ with $s \in \mathbb{Z} / v \mathbb{Z}, t \in(\mathbb{Z} / v \mathbb{Z})^{*}$ then $\widehat{D}$ and $D$ are said to be equivalent.

For projective planes $v$ is equal to $\ell$, which is the total number of points (lines), and $k$ is equal to $q$, which is the number of points on a line and, by duality, of lines through a point. Singer's construction tells us that a point $P_{b}$ and a line $h_{w}$ are incident if and only if

$$
b-w \equiv d_{i} \quad \bmod \ell, \quad i \in \mathbb{Z} / q \mathbb{Z},
$$

where the $q$ elements $d_{i}$ are the elements of a difference set $D$. It is a well known fact that Singer's construction can be extended to projective spaces of higher dimension (see, for instance, [7]), i.e. differences of point indices with the index of the common incident hyperplane build an $(\ell, q, \lambda)$ - difference set. This difference set determines a numbering 
of points depending on hyperplane numbering and viceversa (see Relation (2.8)). Nevertheless, the difference set is not unique since we may have several difference sets with parameters $(\ell, q, \lambda)$ (see [1]) which are equivalent to $D$ or not. We thus fix one difference set $D$ and one ordering of its elements, unique up to cyclic permutations.

According to (2.8) and identifying each point and each hyperplane with its index $b$ or $w$, we choose the local incidence pattern given in Figure $1^{1}$.
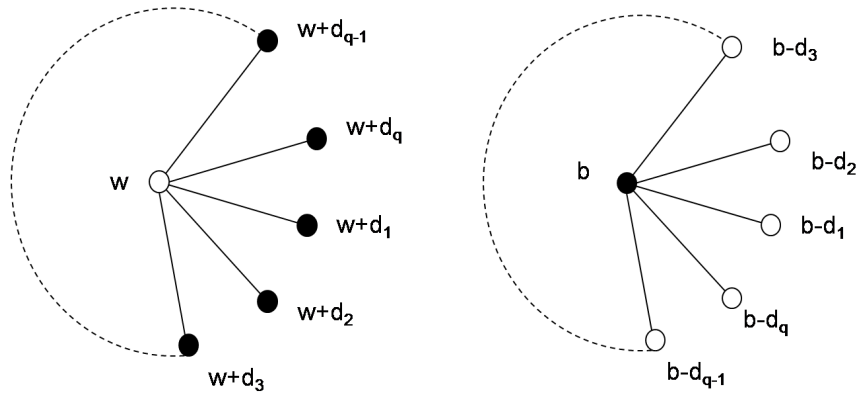

Figure 1: Local incidence pattern.

We construct the embedding of the bipartite graph starting with a white vertex $w$ and with an incident edge $\left(w, w+d_{i}\right)$. Thus according to the local incidence pattern of the black and of the white vertices we have chosen, the next incident edge going clockwise around a cell is $\left(w+d_{i}, w+d_{i}-d_{i+1}\right)$ followed by $\left(w+d_{i}-d_{i+1}, w+2 d_{i}-d_{i+1}\right)$. Repeating this procedure, we obtain a cell boundary with the sequence of edges given in Figure 2.

The construction is applied to successive white vertices until $q \cdot \ell$ different edges of type $\circ-\bullet$ are constructed. Different means here that every two edges differ at least in one of the two indices $b$ or $w$. The construction terminates since all $q \cdot \ell$ incidences between points and hyperplanes are represented by the bipartite graph ${ }^{2}$. For each cell, depending on the value of differences $d_{i}-d_{i+1}$ we reach the starting edge after $2 r$ steps, with $r:=\frac{\ell}{\operatorname{gcd}\left(\left(d_{i}-d_{i+1}\right), \ell\right)}$. Thus we obtain cells with valencies $2 r$ (see Figure 2), where $r$ does not necessarily have the same value for all cells.

\subsection{Wada dessins}

We now consider a special case of the above construction.

Suppose that we may find at least one cyclic ordering of the elements of $D$ such that all differences $d_{i}-d_{i+1}$ are prime to $\ell$. In this case, all cells of the dessin we construct have the same valency $2 \ell$ as it is easy to check. The dessin is therefore uniform with signature

\footnotetext{
${ }^{1}$ We remark here that this is not the only possible incidence pattern we may choose. According to the fixed ordering of the elements of the difference set, the white vertices incident with a black vertex are ordered anticlockwise and the black vertices incident with a white one are ordered clockwise. We have chosen these orderings since we are interested in special uniform dessins called Wada dessins wich we will introduce later on in this section and which are the main topic of study of this paper.

The choice of different orderings is also possible and may give rise to dessins which are not of Wada type (see [12, Chapter 6]).

${ }^{2}$ For reasons of duality between hyperplanes (white vertices) and points (black vertices) we may also carry out the construction starting with edges incident with black vertices. In this case we come to an end when $q \cdot \ell$ different edges of type $\bullet-\circ$ are constructed.
} 


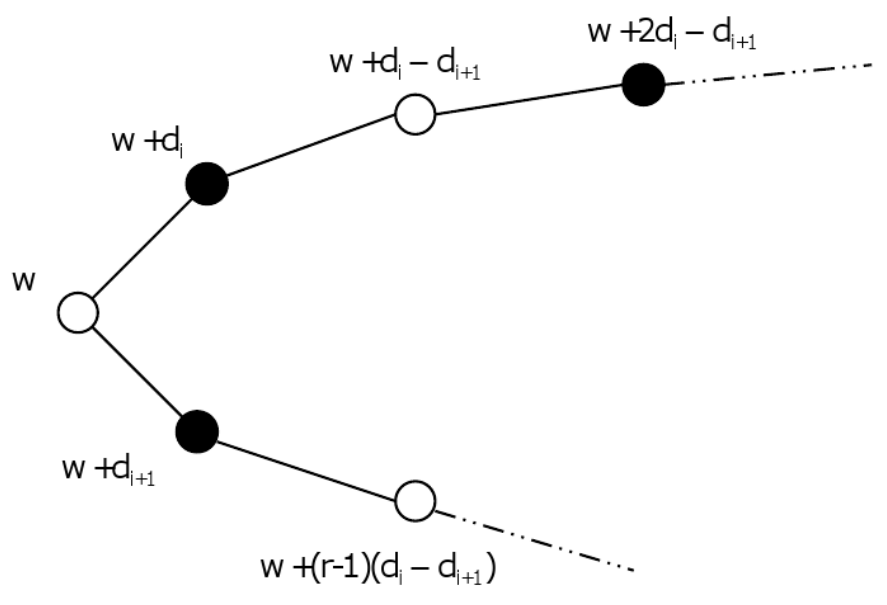

Figure 2: Construction of a cell incident with a white vertex $w$. After $2 r$ steps we reach the starting edge $\left(w, w+d_{i}\right)$.

$(q, q, \ell)$ and with $q$ cells. Such dessins have the following nice property. On the boundary of each cell each white and each black vertex with a given index occurs precisely once. If two white vertices had the same index, then according to the incidence pattern in Figure 1 and to the construction described above we should have

$$
w+\alpha \cdot\left(d_{i}-d_{i+1}\right) \equiv w+\beta \cdot\left(d_{i}-d_{i+1}\right), \quad \alpha, \beta \in \mathbb{Z} / \ell \mathbb{Z},
$$

but this is possible only for $\alpha \equiv \beta \bmod \ell$ since differences $\left(d_{i}-d_{i+1}\right)$ are prime to $\ell$. In a similar way we may prove that it is not possible to have two black vertices with the same index on the same cell boundary.

This property was first described by Streit and Wolfart [14] for bipartite graphs of projective planes $\mathbb{P}^{2}\left(\mathbb{F}_{n}\right)$ and is called Wada property. The choice of the name Wada goes back to the theory of dynamical system and of the Lakes of Wada. It is, in fact, possible to divide the euclidean plane in three regions such that all the points on the boundary of one region are also on the boundary of the other two (see e.g. [4, Chapter 4]). To emphasize the analogy between this phenomenon and the one observed for the cells of their dessins, Streit and Wolfart called them Wada dessins.

More in general, the Wada property may also be observed for embeddings of bipartite graphs associated with projective spaces of higher dimension. By construction, the Wada dessins we obtain are always uniform. Moreover, due to the action of the cyclic group $\Sigma_{\ell}$ on the elements of projective spaces (see Section 2), these dessins have the property that at least the group $\Sigma_{\ell}$ is a group of automorphisms. This group acts transitively on the black and on the white vertices permuting them cyclically on the cell boundaries. This action induces a transitive action on the set of edges of type $\circ-\bullet$ and on the set of edges of type - - o belonging to the boundary of each cell (see [12, Chapter 5] for more details).

We conclude by giving the following definition of Wada compatibility for orderings of elements of difference sets $D$ associated with projective spaces: 
Definition 2.3. We call orderings of the $q$ elements of a difference set $D$ associated with a projective space $\mathbb{P}^{m}\left(\mathbb{F}_{n}\right)$ Wada compatible if differences $\bmod \ell$ of consecutive elements $d_{i}, d_{i+1}$ of $D$ are prime to $\ell$, i.e.:

$$
\operatorname{gcd}\left(\left(d_{i}-d_{i+1}\right), \ell\right)=1 \quad \forall i \in \mathbb{Z} / q \mathbb{Z} .
$$

Remark 2.4. As we have remarked above the difference set $D$ with the chosen element ordering is not unique. Multiplying $D$ with integers $t \in(\mathbb{Z} / \ell \mathbb{Z})^{*}$ and shifting it with integers $s \in \mathbb{Z} / \ell \mathbb{Z}$ we obtain new difference sets equivalent to $D$. If we do not change the element ordering of the original difference set or if we only permute it cyclically, the Wada dessins we construct are isomorphic to each other. However, for non-cyclic permutations and for difference sets with the same parameters $(\ell, q, \lambda)$ but non-equivalent to $D$, even if the chosen ordering is Wada compatible, the dessins we construct are in general not isomorphic to each other. We may, in fact, obtain a different embedding of the graph into an orientable surface and a different Belyı̆ function.

For more general and open questions about if and how many non-equivalent difference sets with the same parameter set exist, see e.g. [1].

\section{The Frobenius Automorphism}

We consider again the identification of the projective space $\mathbb{P}^{m}\left(\mathbb{F}_{n}\right), n=p^{e}$, with the quotient of multiplicative cyclic groups:

$$
\mathbb{P}^{m}\left(\mathbb{F}_{n}\right) \cong \mathbb{F}_{n^{m+1}}^{*} / \mathbb{F}_{n}^{*}
$$

Since $\mathbb{F}_{n^{m+1}}$ is a finite field, we know that there exists an automorphism $\sigma$, the Frobenius automorphism, acting on the elements $a \in \mathbb{F}_{n^{m+1}}$ in the following way:

$$
\begin{aligned}
\sigma: \mathbb{F}_{n^{m+1}} & \longrightarrow \mathbb{F}_{n^{m+1}}, \\
a & \longmapsto a^{p} .
\end{aligned}
$$

The Frobenius automorphism generates the Galois group $\operatorname{Gal}\left(\mathbb{F}_{n^{m+1}} / \mathbb{F}_{p}\right) \cong \Phi_{f}$ as a cyclic group of order $f=e \cdot(m+1)$.

As we have seen in Section 2, we may identify points $P_{b}$ and hyperplanes $h_{w}$ of $\mathbb{P}^{m}\left(\mathbb{F}_{n}\right)$ with powers of a generator of $\mathbb{F}_{n^{m+1}}^{*} / \mathbb{F}_{n}^{*}$ (see Relation (2.5)). Thus each $\sigma^{u} \in \Phi_{f}$, where $u \in \mathbb{Z} / f \mathbb{Z}$, acts on $P_{b}$ and $h_{w}$ as:

$$
\begin{aligned}
\sigma^{u}: P_{b} & \longmapsto P_{b p^{u}}, \\
h_{w} & \longmapsto h_{w p^{u}} .
\end{aligned}
$$

The action of $\Phi_{f}$ subdivides the set of points $\mathcal{P}$ and the set of hyperplanes $\mathcal{H}$ into orbits with lengths $\varphi \in \mathbb{N}, \varphi \mid f$. Each set always contains at least one orbit of length one, i.e. the orbit of the point $P_{0} \in \mathcal{P}$ and the orbit of the hyperplane $h_{0} \in \mathcal{H}$. In fact,

$$
\begin{aligned}
\sigma^{u}: P_{0} & \longmapsto P_{0}, \\
h_{0} & \longmapsto h_{0} \quad \forall u \in \mathbb{Z} / f \mathbb{Z} .
\end{aligned}
$$

\subsection{Frobenius difference sets}

Not every automorphism of projective spaces $\mathbb{P}^{m}\left(\mathbb{F}_{n}\right)$ leads to an automorphism of associated dessins d'enfants. Whether the Frobenius automorphism induces an automorphism 
of associated dessins or not, depends, basically, on the difference set we choose for the construction. First of all, let us look at an example.

Example 3.1. We consider the projective space $\mathbb{P}^{4}\left(\mathbb{F}_{2}\right)$ with parameters $\ell=31$ and $q=$ 15. For the construction of associated dessins we use the difference set $D$ and the shift $D^{\prime} \equiv D-1 \bmod 31$ with their elements ordered in the following way:

$$
\begin{aligned}
D & =\{1,3,15,2,6,30,4,12,29,8,24,27,16,17,23\} \bmod 31 \\
D^{\prime} & \equiv D-1 \quad \bmod 31 \\
& =\{0,2,14,1,5,29,3,11,28,7,23,26,15,16,22\} \quad \bmod 31 .
\end{aligned}
$$

Recalling the incidence pattern given in Figure 1, we construct two dessins $\mathcal{D}$ and $\mathcal{D}^{\prime}$. We are sure that they have the Wada property, since $\ell=31$ is prime. The dessins have signature $(15,15,31)$ and 15 cells. Due to the action on points and on hyperplanes of $\mathbb{P}^{4}\left(\mathbb{F}_{2}\right)$ (see
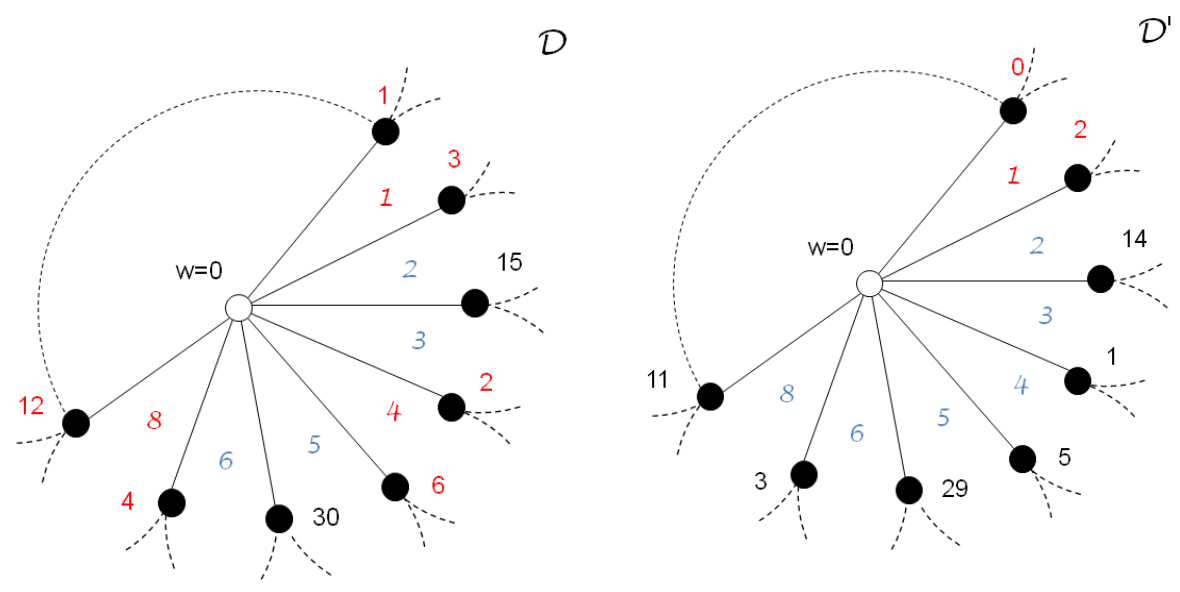

Figure 3: Sketch of dessins $\mathcal{D}$ and $\mathcal{D}^{\prime}$ associated with $\mathbb{P}^{4}\left(\mathbb{F}_{2}\right)$. In red we emphasize the action of the Frobenius automorphism $\sigma$ on the cells. The action of $\sigma$ turns out to be an automorphism of the dessin $\mathcal{D}$ but not of the dessin $\mathcal{D}^{\prime}$ (see text for details).

Relation (3.1)), the Frobenius automorphism $\sigma$ acts on the vertices of each dessin by a multiplication with the prime two:

$$
\begin{aligned}
\sigma: \quad b \longmapsto b \cdot 2, \\
w \longmapsto w \cdot 2 .
\end{aligned}
$$

Lookig at Figure 3 and with an easy check of the action on the vertices, we observe that $\sigma$ is an automorphism of $\mathcal{D}$ but not of $\mathcal{D}^{\prime}$. On $\mathcal{D}$ it rotates the cells around the fixed vertex $w=0$-and by duality also around $b=0$ - by an angle $\omega=\frac{2 \pi}{5}$.

The reason for the different action is due to the different behaviour of the sets $D$ and $D^{\prime}$ under the action of $\sigma$. According to Singer's construction, elements of difference sets associated with projective spaces $\mathbb{P}^{m}\left(\mathbb{F}_{n}\right)$ correspond to differences of indices of points and of incident hyperplanes. Thus, recalling the action of $\sigma$ on points and hyperplanes (see 
Relation (3.1)), its action on the elements of $D$ and $D^{\prime}$ is a multiplication with the integer two. Under such multiplication the set $D$ is fixed up to a cyclic permutation of its elements. On the contrary, the set $D^{\prime}$ is not fixed, as it is easy to check:

$$
\begin{aligned}
& \sigma(D)=\{2,6,30,4,12,29,8,24,27,16,17,23,1,3,15\} \quad \bmod 31 \\
& \sigma\left(D^{\prime}\right)=\{0,4,28,2,10,27,6,22,25,14,15,21,30,1,13\} \quad \bmod 31 .
\end{aligned}
$$

We will analyse more closely the ordering of the elements of $D$ in the next section.

Here we formulate the following

Proposition-Definition 3.2. Let $\mathbb{P}^{m}\left(\mathbb{F}_{n}\right) \cong \mathbb{F}_{n^{m+1}}^{*} / \mathbb{F}_{n}^{*}$ be a projective space, $n=p^{e}, p$ prime, containing $\ell$ points and $\ell$ hyperplanes whose incidence can be described using difference sets. The Frobenius automorphism determines at least one difference set $D_{f}$ fixed under the action of $\Phi_{f} \cong \operatorname{Gal}\left(\mathbb{F}_{n^{m+1}} / \mathbb{F}_{p}\right)$. Multiplying $D_{f}$ with integers $t \in(\mathbb{Z} / \ell \mathbb{Z})^{*}$, we obtain further difference sets fixed under the action of $\Phi_{f}$.

We call $D_{f}$ and all difference sets $t \cdot D_{f}$ Frobenius difference sets.

Proof. As we have seen in Section $3, \Phi_{f}$ divides the set of points $\mathcal{P}$ and the set of hyperplanes $\mathcal{H}$ of $\mathbb{P}^{m}\left(\mathbb{F}_{n}\right)$ into orbits with possibly different lengths. In particular, there is always at least one orbit of length one in each set. In fact, $\Phi_{f}$ always fixes the elements $P_{0} \in \mathcal{P}$ and $h_{0} \in \mathcal{H}$. Let us consider the hyperplane $h_{0}$. Since $\Phi_{f}$ is a group of automorphisms of $\mathbb{P}^{m}\left(\mathbb{F}_{n}\right)$, each $\sigma^{u} \in \Phi_{f}$ preserves incidence. If we now consider the set $\mathcal{P}_{0}=\left\{P_{i_{0}}, P_{i_{1}}, \ldots, P_{i_{q-1}}\right\}$ of points on $h_{0}$, since

$$
\sigma^{u}: h_{0} \longmapsto h_{0} \quad \forall \sigma^{u} \in \Phi_{f},
$$

by incidence preservation we have as well:

$$
\sigma^{u}: \mathcal{P}_{0} \longmapsto \mathcal{P}_{0} \quad \forall \sigma^{u} \in \Phi_{f}
$$

According to Singer's construction, differences of point indices with the index of the common incident hyperplane describe a difference set (see Section 2.1), thus the indices of the points belonging to $\mathcal{P}_{0}$ form the Frobenius difference set $D_{f}$ we are looking for. It is easy to see that multiplying $D_{f}$ with integers $t \in(\mathbb{Z} / \ell \mathbb{Z})^{*}$ we still obtain Frobenius difference sets. In fact, $\Phi_{f}$ acts on the elements of $D_{f}$ and of each $t \cdot D_{f}$ by multiplication with powers $p^{u}, u \in \mathbb{Z} / f \mathbb{Z}$, so we have:

$$
p^{u} \cdot t \cdot D_{f}=t \cdot p^{u} \cdot D_{f} \equiv t \cdot D_{f} \quad \bmod \ell \quad \forall t \in(\mathbb{Z} / \ell \mathbb{Z})^{*},
$$

i.e. each $t \cdot D_{f}$ is fixed under the action of $\Phi_{f}$.

Remark 3.3. The difference set $D_{f}$ and the sets $t \cdot D_{f}$ determined by $\Phi_{f}$ may not be unique. In fact, as we have already remarked in Sections 2.1 and 2.2, other difference sets $D_{f}$ with parameters $(\ell, q, \lambda)$ may exist which are equivalent or non-equivalent to $D_{f}$ and are fixed by $\Phi_{f}$. The case of difference sets resulting from shifts of $D_{f}$ will be analysed more closely later on in this section.

The above proof implies 
Corollary 3.4. The cyclic group $\Phi_{f}$ acts on the elements of a Frobenius difference set $D_{f}$ by a multiplication with powers $p^{u}, u \in \mathbb{Z} / f \mathbb{Z}$ and we have

$$
p^{u} D_{f} \equiv D_{f} \quad \bmod \ell \quad \forall u \in(\mathbb{Z} / f \mathbb{Z}) .
$$

Due to the action of $\Phi_{f}$ on points and hyperplanes, from the above proof it also follows that $\Phi_{f}$ divides the points on $h_{0}$ into orbits with lengths $\varphi, \varphi \in \mathbb{N}, \varphi \mid f$. As $D_{f}$ consists of the indices of these points, this means that $\Phi_{f}$ also divides the elements of $D_{f}$ into orbits with lengths $\varphi$. Hence, we formulate the following

Corollary 3.5. Under the action of $\Phi_{f}$, the elements of a Frobenius difference set $D_{f}$ are subdivided into orbits with lengths $\varphi, \varphi \in \mathbb{N}, \varphi \mid f$. These orbits correspond to orbits of points in $\mathbb{P}^{m}\left(\mathbb{F}_{n}\right)$.

Example 3.6. In the above example for $\mathbb{P}^{4}\left(\mathbb{F}_{2}\right)$ the Frobenius automorphism generates the Galois group $\Phi_{5} \cong \operatorname{Gal}\left(\mathbb{F}_{2}^{5} / \mathbb{F}_{2}\right)$. This group acts on the points $P_{i}$ and on the hyperplanes $h_{i}$ of $\mathbb{P}^{4}\left(\mathbb{F}_{2}\right)$ by a multiplication with powers $2^{u}, u \in \mathbb{Z} / 5 \mathbb{Z}$ :

$$
\begin{aligned}
\sigma^{u} \in \Phi_{5}: \quad & P_{i} \longmapsto P_{i 2^{u}}, \\
& h_{i} \longmapsto h_{i 2^{u}}, \quad u \in(\mathbb{Z} / 5 \mathbb{Z}) .
\end{aligned}
$$

The elements of the difference set $D_{5}$ may be identified with the indices of the 15 points on the fixed hyperplane $h_{0}$. The difference set $D_{5}$ is fixed under the action of $\sigma$ and therefore of $\Phi_{5}$. Its elements are subdivided by $\Phi_{5}$ into three orbits of length five:

$$
\{1,2,4,8,16\}, \quad\{3,6,12,24,17\}, \quad\{15,30,29,27,23\} .
$$

Example 3.7. Let us consider the projective space $\mathbb{P}^{3}\left(\mathbb{F}_{3}\right) \cong \mathbb{F}_{3^{4}}^{*} / \mathbb{F}_{3}^{*}$ with 40 points and, by duality, with 40 hyperplanes. The Frobenius automorphism $\sigma$ generates the Galois group $\Phi_{4} \cong \operatorname{Gal}\left(\mathbb{F}_{3^{4}} / \mathbb{F}_{3}\right)$ which acts on the points $P_{i}$ and on the hyperplanes $h_{i}$ as

$$
\begin{aligned}
\sigma^{u} \in \Phi_{4}: & P_{i} \longmapsto P_{i 3^{u}}, \\
& h_{i} \longmapsto h_{i 3^{u}}, \quad u \in(\mathbb{Z} / 4 \mathbb{Z}) .
\end{aligned}
$$

The elements of the following $(40,13,4)$-difference set ([1]) may be identified with the indices of the 13 points on the hyperplane $h_{0}$ fixed by $\Phi_{4}$ :

$$
D_{4}=\{21,22,23,25,26,29,34,35,38,0,5,7,15\} \bmod 40 .
$$

The set $D_{4}$ is a Frobenius difference set and it is easy to prove that multiplying it with powers $3^{u}$ we only have a permutation of its elements, i.e. we have

$$
3^{u} D_{4} \equiv D_{4} \quad \bmod 40 \quad \forall u \in(\mathbb{Z} / 4 \mathbb{Z})
$$

The cyclic group $\Phi_{4}$ subdivides the elements of $D_{4}$ into the following five orbits:

$$
\{21,23,29,7\},\{22,26,34,38\},\{25,35\},\{5,15\},\{0\} .
$$

We have seen that difference sets which result from multiplications of Frobenius difference sets $D_{f}$ by elements $t \in(\mathbb{Z} / \ell \mathbb{Z})^{*}$ are still Frobenius difference sets fixed by $\Phi_{f}$. Therefore, it is reasonable to ask about shifts. Indeed, depending on the number of fixed points and of fixed hyperplanes of $\Phi_{f}$ we can have more than one Frobenius difference set: 
Proposition 3.8. Let $\mathbb{P}^{m}\left(\mathbb{F}_{n}\right) \cong \mathbb{F}_{n^{m+1}}^{*} / \mathbb{F}_{n}^{*}$ be a projective space, $n=p^{e}$, p prime. The projective space $\mathbb{P}^{m}\left(\mathbb{F}_{n}\right)$ contains $\ell$ points and $\ell$ hyperplanes whose incidence can be described using a Frobenius difference set $D_{f}$. No shifts of $D_{f}$ are allowed if and only if only $h_{0}$ and equivalently only $P_{0}$ are fixed by $\Phi_{f} \cong \operatorname{Gal}\left(\mathbb{F}_{n^{m+1}} / \mathbb{F}_{p}\right)$. If there are more elements fixed, we will have shifts of $D_{f}$ which are still Frobenius difference sets and:

$$
\text { \#Frobenius difference sets as shifts of } D_{f}=\text { \# fixed hyperplanes (fixed points) of } \Phi_{f} \text {. }
$$

Proof. Let us assume that no shifts of $D_{f}$ are allowed but there is at least another hyperplane $h_{s}$ fixed by $\Phi_{f}$. This would mean that $h_{0}$ and $h_{s}$ share the same point set $\mathcal{P}_{0}$. Nevertheless, sharing the same point set means $h_{0}=h_{s}$.

Now we assume that only the hyperplane $h_{0}$ is fixed by $\Phi_{f}$, but there are two difference sets fixed: the Frobenius difference set $D_{f}$ and a shift of it $D_{f}^{\prime} \equiv\left(D_{f}+s\right) \bmod \ell$. Both $D_{f}$ and $D_{f}^{\prime}$ are defined up to multiplication with elements $t \in(\mathbb{Z} / \ell \mathbb{Z})^{*}$. According to Singer's construction if we identify the points on $h_{0}$ with the elements of $D_{f}$, then we may identify the points on $h_{s}$ with the elements of $D_{f}^{\prime} \equiv\left(D_{f}+s\right) \bmod \ell$. As $D_{f}^{\prime}$ is fixed under the action of $\Phi_{f}$ so should also $h_{s}$ be, but this would be a contradiction to the fact that $h_{0}$ is unique. It thus follows that no shifts of $D_{f}$ are allowed.

As we have already seen, the indices of the points on every hyperplane $h_{s}, s \in \mathbb{Z} / \ell \mathbb{Z}$ can be identified with the elements of a shift $D_{f}+s$. If $\Phi_{f}$ fixes some of the hyperplanes $h_{s}$, it directly follows that the indices of the points on each fixed $h_{s}$ describe Frobenius difference sets $D_{f}+s$ and we have:

\#Frobenius difference sets as shifts of $D_{f}=\#$ fixed hyperplanes (fixed points) of $\Phi_{f}$.

Remark 3.9. From the proof of Proposition 3.8 follows that, once we know a Frobenius difference set $D_{f}$, it is easy to determine other equivalent difference sets fixed by $\Phi_{f}$. We only need to know which of the hyperplanes $h_{s}$ is fixed by $\Phi_{f}$. The corresponding difference set is then $D_{f}+s$.

Example 3.10. For the projective space $\mathbb{P}^{4}\left(\mathbb{F}_{2}\right)$ there are only one hyperplane $h_{0}$ and one point $P_{0}$ fixed under the action of $\Phi_{5}$. It follows that the Frobenius difference set

$$
D_{5}=\{1,3,15,2,6,30,4,12,29,8,24,27,16,17,23\} \quad \bmod 31
$$

is unique up to multiplication with elements $t \in(\mathbb{Z} / 31 \mathbb{Z})^{*}$.

Example 3.11. For the projective space $\mathbb{P}^{3}\left(\mathbb{F}_{3}\right)$ there are two hyperplanes and, by duality, two points fixed by $\Phi_{4}$. The fixed hyperplanes are $h_{0}$ and $h_{20}$ and the fixed points are $P_{0}$ and $P_{20}$. As we have seen in Example 3.7, we may choose

$$
D_{4}=\{21,22,23,25,26,29,34,35,38,0,5,7,15\} \quad \bmod 40
$$

as the Frobenius difference set corresponding to the set of points on $h_{0}$. We, therefore, have that

$$
D_{4}^{\prime} \equiv D_{4}+20 \bmod 40=\{1,2,3,5,6,9,14,15,18,20,25,27,35\} \quad \bmod 40
$$

is the Frobenius difference set corresponding to the set of points on $h_{20}$ and it is easy to prove that $3^{u}\left(D_{4}+20\right) \equiv\left(D_{4}+20\right) \bmod 40$ for all $u \in(\mathbb{Z} / 4 \mathbb{Z})$. 

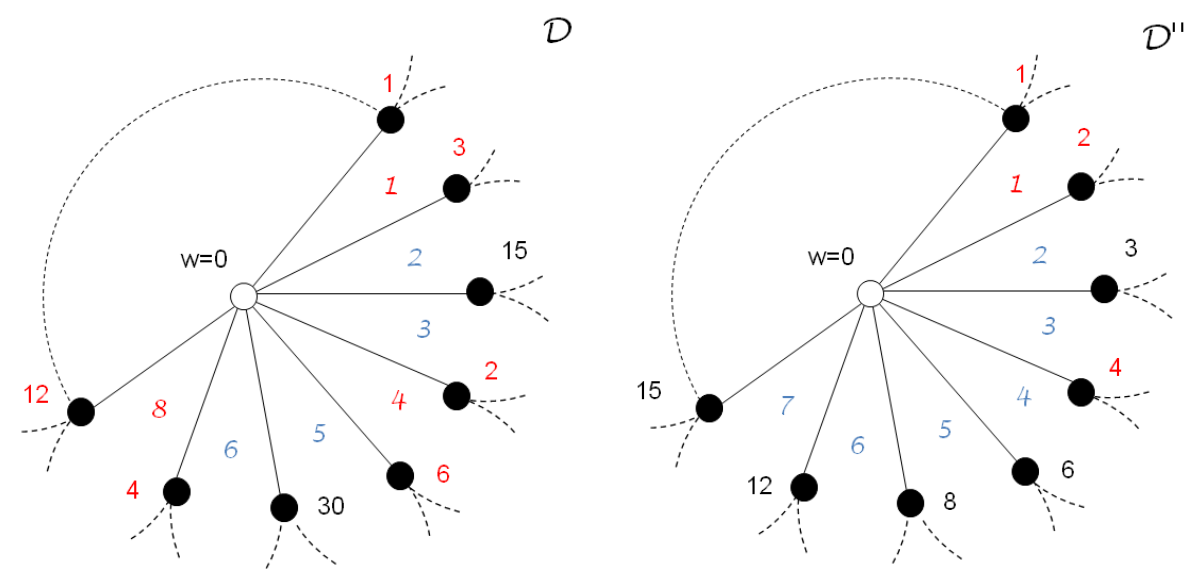

Figure 4: Sketch of dessins $\mathcal{D}$ and $\mathcal{D}^{\prime \prime}$ associated with $\mathbb{P}^{4}\left(\mathbb{F}_{2}\right)$. As in Figure 3 we emphasize in red the action of the Frobenius automorphism on the cells (see text for more details).

\subsection{Frobenius compatibility}

The existence of Frobenius difference sets is a necessary but still not a sufficient condition for the construction of dessins having the Frobenius automorphism as a dessin automorphism. We consider the following example:

Example 3.12. In addition to the dessins $\mathcal{D}$ and $\mathcal{D}^{\prime}$ we have considered in Example 3.1 we construct a third dessin $\mathcal{D}^{\prime \prime}$ associated with the projective space $\mathbb{P}^{4}\left(\mathbb{F}_{2}\right)$. For the construction we use the difference set $D^{\prime \prime}$ whose elements are the elements of $D$ but with a different ordering ${ }^{3}$ :

$$
\begin{gathered}
D=\{1,3,15,2,6,30,4,12,29,8,24,27,16,17,23\} \quad \bmod 31 \\
D^{\prime \prime}=\{1,2,3,4,6,8,12,15,16,17,23,24,27,29,30\} \quad \bmod 31 .
\end{gathered}
$$

According to the incidence pattern given in Figure 1 we may construct the Wada dessin $\mathcal{D}^{\prime \prime}$ sketched in Figure 4. In Example 3.1 we have seen that $\sigma$ is an automorphism of $\mathcal{D}$ acting with a rotation of the cells around the white vertex $w=0$. However, for $\mathcal{D}^{\prime \prime}$ we observe that it is not an automorphism. The different behaviour of the Frobenius automorphism on the dessins $\mathcal{D}$ and $\mathcal{D}^{\prime \prime}$ depends on the ordering of the elements of the difference sets. We consider orbits of elements under the action of the cyclic group $\Phi_{5}$ generated by $\sigma$. As we have seen in Example 3.6 the elements of $D$ are subdivided into three orbits of length five:

$$
\{1,2,4,8,16\}, \quad\{3,6,12,24,17\}, \quad\{15,30,29,27,23\} .
$$

Thus, we are able to construct five blocks: the first block only contains the first elements of each orbit, the second block the second elements of each orbit, etc. The Frobenius automorphism maps the elements of each block onto the elements of the next block. The

\footnotetext{
${ }^{3}$ We use here again the notation $D$ for the difference set $D_{5}$ in order to be consistent with the notation used in Example 3.1.
} 
element ordering of $D$ corresponds to this subdivision into blocks, the element ordering of $D^{\prime \prime}$ does not.

We call the ordering of the elements of $D$ given in the above example Frobenius compatible.

According to the action of $\Phi_{f}$ on the elements of a Frobenius difference set $D_{f}$ (see Corollary 3.4), we give the following definition:

Definition 3.13. Consider the following cyclic orderings of the $q$ elements of a Frobenius difference set $D_{f}$ :

$$
\begin{aligned}
D_{f}= & \left\{d_{1}, \ldots, d_{k}, p^{j} d_{1}, \ldots, p^{j} d_{k}, \ldots \ldots, p^{(f-1) j} d_{1}, \ldots, p^{(f-1) j} d_{k}\right\}, \\
& \text { for some } j \in(\mathbb{Z} / f \mathbb{Z})^{*}, \frac{q}{f}=k .
\end{aligned}
$$

We call such cyclic orderings Frobenius compatible orderings.

Since Wada compatibility is also necessary for the construction of Wada dessins (see Definition 2.3) we formulate moreover

Proposition 3.14. The elements of a Frobenius difference set $D_{f}$ ordered in a Frobenius compatible way are also ordered in a Wada compatible way iff differences of consecutive elements belonging to the subset $\left\{d_{1}, \ldots, d_{k}\right\}$ are prime to $\ell$

$$
\operatorname{gcd}\left(d_{i}-d_{i+1}, \ell\right)=1 \quad \forall i \in\{1, \ldots,(k-1)\}
$$

and iff

$$
\operatorname{gcd}\left(d_{k}-p^{j} d_{1}, \ell\right)=1 .
$$

Proof. If the elements of $D_{f}$ ordered in a Frobenius compatible way also satisfy the Wada condition, then conditions (3.4) and (3.5) necessarily hold.

If condition (3.4) holds then we also have

$$
\operatorname{gcd}\left(p^{j}\left(d_{i}-d_{i+1}\right), \ell\right)=1 \quad \forall j \in(\mathbb{Z} / f \mathbb{Z})^{*} .
$$

In fact, since $\ell=\frac{n^{m+1}-1}{n-1}=n^{m}+n^{m-1}+\cdots+1$ with $n=p^{e}$ we have $\operatorname{gcd}(\ell, p)=1$. This means that all differences of consecutive elements belonging to the subset $\left\{p^{j} d_{1}, \ldots, p^{j} d_{k}\right\}$ are prime to $\ell$. Of course, this may be extended to each other subset $\left\{p^{h \cdot j} d_{1}, \ldots, p^{h \cdot j} d_{k}\right\}$, $h \in \mathbb{Z} / f \mathbb{Z}$.

Condition (3.5) is necessary to make sure that when passing from one subset to the next the Wada condition is also satisfied. If (3.5) holds then for the same reasons as in (3.6) we also have:

$$
\operatorname{gcd}\left(\left(p^{h \cdot j} d_{k}-p^{(h+1) \cdot j} d_{1}\right), \ell\right)=1 \quad \forall h \in \mathbb{Z} / f \mathbb{Z} .
$$

We proceed considering Wada dessins constructed with Frobenius difference sets whose elements are ordered in a Frobenius compatible way. For these dessins the cyclic group $\Phi_{f}$ is a group of automorphisms. We examine this fact more in detail in the next sections. 


\section{A 'nice' case}

In Section 3.1 we have seen that for each projective space $\mathbb{P}^{m}\left(\mathbb{F}_{n}\right)$ there always exists at least one Frobenius difference set $D_{f}$. Unfortunately, we cannot always order its elements in a Frobenius compatible way. In fact, it may happen that the cyclic group $\Phi_{f}$ divides the elements of $D_{f}$ into orbits with different lenghts. In Example 3.7 we have seen that the cyclic group $\Phi_{4}$ divides the elements of the difference set $D_{4}$ associated with $\mathbb{P}^{3}\left(\mathbb{F}_{3}\right)$ into orbits with lenghts four, two and one. In general, it is not easy to determine the necessary conditions for the parameters $m$ and $n$ of $\mathbb{P}^{m}\left(\mathbb{F}_{n}\right)$ so that we can predict the existence of Frobenius compatible orderings. Here we consider a 'nice' case and we see that if the order of the cyclic group $\Phi_{f}$ is prime, under some conditions, Frobenius compatible orderings exist.

We first formulate two lemmas:

Lemma 4.1. Let $f$ be the order of the cyclic group $\Phi_{f} \cong G a l\left(\mathbb{F}_{n^{m+1}} / \mathbb{F}_{p}\right)$ generated by the Frobenius automorphism acting on a projective space $\mathbb{P}^{m}\left(\mathbb{F}_{n}\right)$ with $n=p^{e}, e \in \mathbb{N} \backslash\{0\}$. Let $f$ be prime, thus we have:

$$
n=p, \quad f=m+1, \quad \Phi_{f} \cong \operatorname{Gal}\left(\mathbb{F}_{p^{m+1}} / \mathbb{F}_{p}\right) .
$$

If $p \neq m+1$ and $p \not \equiv 1 \bmod (m+1)$, then $f$ divides the valency $q$ of the white and of the black vertices.

Proof. The proof follows from Fermat's little theorem.

For $n=p$ the integer $q$ is given by (recall Relation (2.3)):

$$
q=\frac{p^{m}-1}{p-1}
$$

The integer $f=m+1$ divides $q$ if

$$
p^{m} \equiv 1 \bmod (m+1),
$$

and this is true due to Fermat's little theorem since we have chosen $p \neq m+1$. Now we need the denominator not to 'destroy' the divisibility property. In fact, we have to choose

$$
p \not \equiv 1 \bmod (m+1) \text {, }
$$

since for $p \equiv 1 \bmod (m+1)$ we obtain:

$$
q=p^{m-1}+\cdots+1 \equiv m \bmod (m+1)
$$

and $\operatorname{gcd}(m,(m+1))=1$.

Lemma 4.2. We consider the Frobenius difference set $D_{f}$ fixed by the cyclic group $\Phi_{f}$. Under the conditions of Lemma 4.1 on $f$ and $n$, no shifts of $D_{f}$ are fixed by $\Phi_{f}$ and the following properties hold:

1. $0 \notin D_{f}$ and

2. all $\Phi_{f}$-orbits of elements $d_{i} \in D_{f}$ have length $f$. 
Proof. As we have seen in Corollary 3.4, the group $\Phi_{f}$ acts on the elements of $D_{f}$ by multiplication with powers of $p$ and it divides the elements into orbits whose lengths are divisors of $f$. Since $f$ is prime, we will only have orbits of length 1 or of length $f$. Suppose we have at least one orbit of length one. Since $f$ divides $q$ (see Lemma 4.1 above), we necessarily have at least $f-1$ other orbits of length 1 . Let $0 \in D_{f}$ and let $\{0\}$ be an orbit of length one.

Recalling some ideas of Baumert [1] about projective planes, we first show that $\operatorname{gcd}(p-$ $1, \ell)=1$.

Dividing $\ell$ by $p-1$ we obtain:

$$
\ell=(p-1)\left(p^{m-1}+\cdots+(m-1) p+m\right)+(m+1) .
$$

We know

$$
\operatorname{gcd}(p-1, m+1)=1
$$

due to $p \not \equiv 1 \bmod (m+1)$ and to $m+1$ being prime. From the Euclidean algorithm it therefore follows that

$$
\operatorname{gcd}(p-1, \ell)=1 .
$$

Let us suppose that there exists another Frobenius difference set $D_{f}^{\prime} \equiv\left(D_{f}+s\right) \bmod \ell$ fixed by $\Phi_{f}$. For the integer $s \in \mathbb{Z} / \ell \mathbb{Z}$ we have:

$$
p \cdot s \equiv s \quad \bmod \ell \quad \text { i.e. }(p-1) \cdot s \equiv 0 \bmod \ell .
$$

Since $\operatorname{gcd}(p-1, \ell)=1$, equation (4.2) can be satisfied only for $s \equiv 0 \bmod \ell$. Thus no shifts of $D_{f}$ are allowed.

Now, if $(f-1)$ elements $d_{i} \in D_{f}$ with $d_{i} \not \equiv 0 \bmod \ell$ are fixed by $p$, then they should satisfy the congruence relation:

$$
p \cdot d_{i} \equiv d_{i} \bmod \ell \Longrightarrow(p-1) \cdot d_{i} \equiv 0 \bmod \ell .
$$

Again, since $\operatorname{gcd}(p-1, \ell)=1$, this congruence can be satisfied only for $d_{i} \equiv 0 \bmod \ell$. This means that $\{0\}$ is the only possible orbit of length one under the action of $p$. Nevertheless, we must exclude it since in this case $f$ would not divide $q$.

It follows that $0 \notin D_{f}$ and that $D_{f}$ is decomposed only into $\Phi_{f}$-orbits of length $f$.

Thanks to the above lemma we may choose cyclic orderings of the elements of $D_{f}$ as we have defined them in 3.13. We have

Corollary 4.3. Under the conditions of Lemma 4.1, the elements of a Frobenius difference set $D_{f}$ fixed by the cyclic group $\Phi_{f}$ may be ordered in a Frobenius compatible way. This ordering is fixed under the action of $\Phi_{f}$ up to cyclic permutations.

Using the Frobenius difference set $D_{f}$ with Frobenius compatible element orderings we construct dessins associated with projective spaces. If the dessins have the Wada property, then the cyclic group $\Phi_{f}$ is a group of automorphisms.

Proposition 4.4. Let $\Phi_{f}$ be the cyclic group generated by the Frobenius automorphism acting on a finite projective space $\mathbb{P}^{m}\left(\mathbb{F}_{n}\right), n=p^{e}$. Let $f$ be prime and $p \neq m+1$, $p \not \equiv 1 \bmod (m+1)$. Let $D_{f}$ be a Frobenius difference set fixed by $\Phi_{f}$ whose elements are ordered in a Frobenius compatible way. If the elements of $D_{f}$ are also ordered in a Wada compatible way so that we can construct a $(q, q, \ell)$-Wada dessin $\mathcal{D}$, then $\Phi_{f}$ is a group of automorphisms of $\mathcal{D}$ acting freely on the edges and rotating the set of cells around the vertices $b=w=0$ fixed by $\Phi_{f}$. 

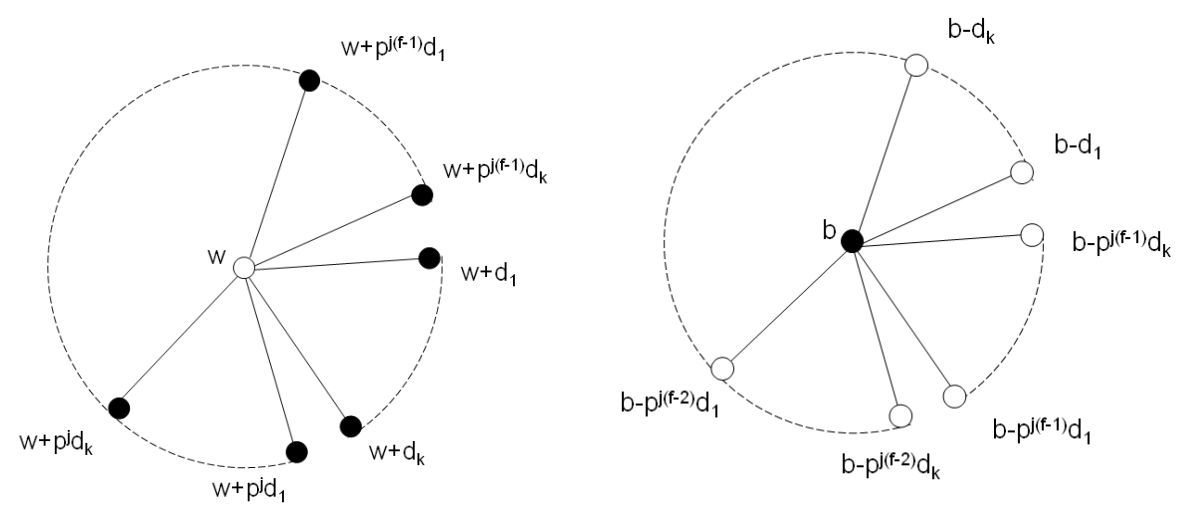

Figure 5: Local incidence patterns with Frobenius compatible ordering of the elements of $D_{f}$.

Proof. We suppose that at least one Frobenius compatible ordering of the elements of $D_{f}$ considered up to cyclic permutations is also Wada compatible and we construct a $(q, q, \ell)$ Wada dessin $\mathcal{D}$. Since $f \mid q$ (see Lemma 4.1 above), the group $\Phi_{f}$ has a suitable size to be a group of automorphisms of $\mathcal{D}$ acting on the $q$ cells of $\mathcal{D}$. The cyclic group $\Phi_{f}$ is generated by the Frobenius automorphism $\sigma$. We consider the action of $\sigma$ on the points and hyperplanes of $\mathbb{P}^{m}\left(\mathbb{F}_{n}\right)$ and therefore on the vertices of $\mathcal{D}$. As we have seen in Section 3 it acts by multiplication of the indices with the prime $p$ :

$$
\begin{aligned}
\sigma: P_{b} & \longmapsto P_{b p}, \\
h_{w} & \longmapsto h_{w p} .
\end{aligned}
$$

According to the notation introduced in Definition 3.13, we write the edges of $\mathcal{D}$ as $e_{\nu, i}^{w}=\left(w, w+p^{\nu} d_{i}\right) \quad(\circ-\bullet) \quad$ and $\quad e_{\nu, i}^{b}=\left(b, b-p^{\nu} d_{i}\right) \quad(\bullet-\circ)$ with $\nu \in \mathbb{Z} / f \mathbb{Z}$, $i \in\{1, \ldots, k\}$. The action of $\sigma$ on the edges is given by:

$$
\begin{gathered}
\sigma: e_{\nu, i}^{w}=\left(w, w+p^{\nu} d_{i}\right) \longmapsto p \cdot e_{\nu, i}^{w}=\left(p \cdot w, p\left(w+p^{\nu} d_{i}\right)\right), \\
e_{\nu, i}^{b}=\left(b, b-p^{\nu} d_{i}\right) \longmapsto p \cdot e_{\nu, i}^{b}=\left(p \cdot b, p\left(b-p^{\nu} d_{i}\right)\right) .
\end{gathered}
$$

If $w$ and $b$ are not fixed under the action of $\Phi_{f}$, then none of the edges $e_{\nu, i}^{w}$ and $e_{\nu, i}^{b}$ is fixed by $\sigma$. If, on the contrary, $w$ and $b$ are fixed, then $\sigma\left(e_{\nu, i}^{w}\right) \neq e_{\nu, i}^{w}$ and $\sigma\left(e_{\nu, i}^{b}\right) \neq e_{\nu, i}^{b}$ only if $d_{i}$ is not fixed by $p$. Indeed, this is true, otherwise we could not have chosen $D_{f}$ with a Frobenius compatible ordering of its elements. Thus $\Phi_{f}$ does not fix any of the edges and we say that its action is free on them.

We now consider the action of $\Phi_{f}$ on the cells around the vertices $w$ and $b$ of $\mathcal{D}$ which are fixed by $\Phi_{f}$. Since $\Phi_{f}$ only fixes the difference set $D_{f}$ (see Lemma 4.2), this means that it only fixes the vertices $b=w=0$ of the dessin. In fact, according to Singer's construction (see Section 2.1), elements of difference sets $D$ associated with projective spaces correspond to indices of points on hyperplanes (and by duality of hyperplanes through points). As $D_{f}$ is the only difference set fixed by $\Phi_{f}$, only $h_{0}$ and $P_{0}$ are fixed by $\Phi_{f}$, from which it follows that $w=b=0$ are the only vertices fixed by $\Phi_{f}$. 
Recall the incidence pattern of each vertex given in Figure 5 where the elements of $D_{f}$ are ordered in a Frobenius compatible way. The incidence pattern is fixed up to cyclic permutations of the elements of $D_{f}$. The action of $\sigma$ on the cells $\mathcal{C}_{c}, c \in \mathbb{Z} / q \mathbb{Z}$ around $w=0$ results in a mapping of every cell to a following cell (see Figure 6) such that:

$$
\begin{aligned}
& \sigma: \mathcal{C}_{c} \longmapsto \mathcal{C}_{c+m k} \quad \bmod q, \\
& c \in \mathbb{Z} / q \mathbb{Z}, m \in \mathbb{Z} / f \mathbb{Z}, k=\frac{q}{f} .
\end{aligned}
$$

We obtain the following relation between powers of $p$ and the cells we run through:

\begin{tabular}{r|l}
$p$-powers & cells \\
\hline$p^{0}$ & $\mathcal{C}_{c}$ \\
$p^{1}$ & $\mathcal{C}_{c+m k} \quad \bmod q$ \\
$p^{2}$ & $\mathcal{C}_{c+2 m k} \bmod q$ \\
$p^{3}$ & $\mathcal{C}_{c+3 m k} \bmod q$ \\
$\vdots$ & $\vdots$ \\
$p^{f-1}$ & $\mathcal{C}_{c+(f-1) m k} \bmod q$
\end{tabular}

Evidently, $\sigma$ describes a rotation of the set of cells around 0 . The order of the rotation is $f$.

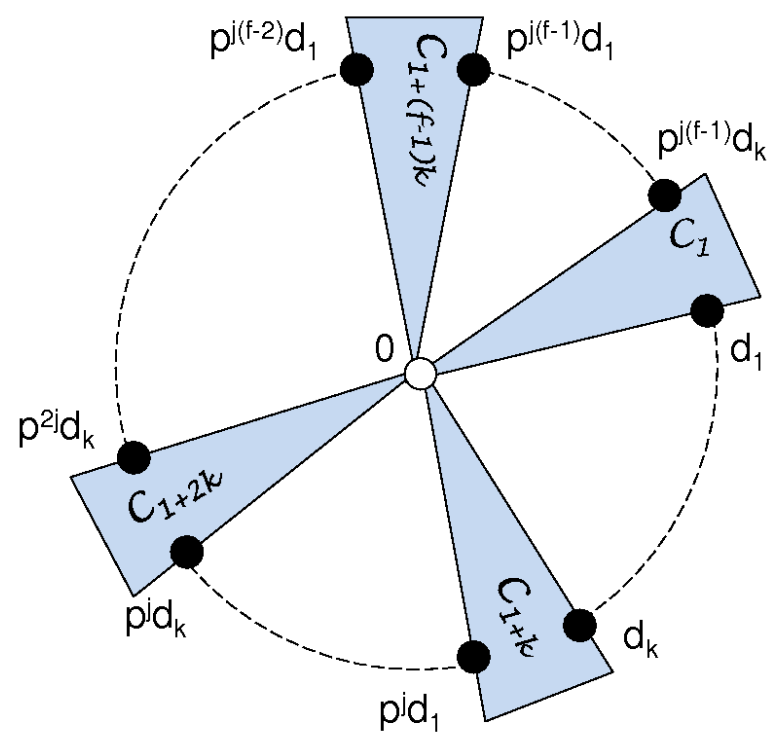

Figure 6: Local ordering of the cells around $w=0$ with a Frobenius difference set $D_{f}$. The elements of $D_{f}$ are ordered in a Frobenius compatible way. 
Remark 4.5. 1. A consequence of the above proof is that $\Phi_{f}$ acts freely not only on the edges but also on the cells and it divides them into $k$ orbits of length $f$.

2. We consider the vertices fixed by $\Phi_{f}$ as fixed points $x$ on the target surface $X$ of the embedding. Then, introducing local coordinates $z$ we may suppose that $z(x)=0$ and that $\Phi_{f}$ acts on a neighbourhood of $z=0$ by multiplication with powers of a root of unity $\zeta_{f}$ :

$$
z \longmapsto \zeta_{f}^{a} z, \quad a \in \mathbb{Z} / f \mathbb{Z} .
$$

The root of unity $\zeta_{f}$ is called multiplier of the automorphism $\sigma \in \Phi_{f}$ on $X$ (see [14]).

Example 4.6. For the projective space $\mathbb{P}^{4}\left(\mathbb{F}_{2}\right)$, we have considered in several of the above examples, we have $f=m+1=5$ and $p \neq 5, p \not \equiv 1 \bmod 5$, thus the conditions of Proposition 4.4 are satisfied. We construct a $(15,15,31)$-Wada dessin with the Frobenius difference set:

$$
D_{5}=\{1,3,15,2,6,30,4,12,29,8,24,27,16,17,23\} \quad \bmod 31
$$

whose elements are ordered in a Frobenius compatible way (see Example 3.1 and 3.12). The cyclic group $\Phi_{5} \cong \operatorname{Gal}\left(\mathbb{F}_{2^{5}} / \mathbb{F}_{2}\right)$, generated by the Frobenius automorphism, acts freely on the edges and on the cells. On the cells it acts with a rotation by the angle $\omega=\frac{2 \pi}{5}$ around the fixed vertices $b=w=0$. The cells are subdivided into three orbits of length five.

Other dessins, whose parameters satisfy the conditions of Proposition 4.4, are given in Table 2. For most of the projective spaces listed there $\ell$ is prime, so we are sure we may construct Wada dessins regardless of the chosen element orderings of the associated Frobenius difference set.

For the spaces $\mathbb{P}^{4}\left(\mathbb{F}_{3}\right)$ and $\mathbb{P}^{10}\left(\mathbb{F}_{2}\right)$ the integer $\ell$ is not prime, thus we have to check whether Frobenius compatible orderings of elements are also Wada compatible. According to Proposition 3.14, we only need to check differences of the first block of elements and the one difference at the 'transition' between the first and the second block. Other differences are multiplications with powers of the prime $p$ for which we have $\operatorname{gcd}(\ell, p)=1$.

For both spaces the prime factors of the integer $\ell$ are quite big. In fact, we have $\ell=$ $121=11 \cdot 11$ and $\ell=2047=89 \cdot 23$, so it is very likely to find Frobenius and Wada compatible orderings. For instance, for $\mathbb{P}^{4}\left(\mathbb{F}_{3}\right)$ it is easy to check that the ordering of the elements of the following Frobenius difference set is Frobenius and Wada compatible.

$$
\begin{aligned}
D_{5}=\{1,4,7,11,13,34,25,67,3,12,21,33,39,102,75,80,9,36,63,99,117,64,104, & \\
& 119,27,108,68,55,109,71,70,115,81,82,83,44,85,92,89,103\} \quad \bmod 121 .
\end{aligned}
$$

In this case, the group generated by the Frobenius automorphism is cyclic of order five and it divides the elements of $D_{5}$ into eight orbits of length five.

\section{Concluding remarks}

We consider projective spaces $\mathbb{P}^{m}\left(\mathbb{F}_{n}\right)$ with general $m$ and $n$, which do not necessarily satisfy the conditions of Proposition 4.4. In this case, it is more difficult to predict whether 


\begin{tabular}{c|c|c|c|c|c}
\hline & $q$ & $\ell$ & $f$ & Wada & Frobenius \\
\hline $\mathbb{P}^{2}\left(\mathbb{F}_{5}\right)$ & 6 & 31 & 3 & $(6,6,31)$ & $\Phi_{3} \cong \mathrm{Gal}\left(\mathbb{F}_{5^{3}} / \mathbb{F}_{5}\right)$ \\
$\mathbb{P}^{4}\left(\mathbb{F}_{2}\right)$ & 15 & 31 & 5 & $(15,15,31)$ & $\Phi_{5} \cong \mathrm{Gal}\left(\mathbb{F}_{2^{5}} / \mathbb{F}_{2}\right)$ \\
$\mathbb{P}^{4}\left(\mathbb{F}_{3}\right)$ & 40 & $121=11^{2}$ & 5 & $(40,40,121)$ & $\Phi_{5} \cong \mathrm{Gal}\left(\mathbb{F}_{3^{5}} / \mathbb{F}_{3}\right)$ \\
$\mathbb{P}^{4}\left(\mathbb{F}_{7}\right)$ & 400 & 2801 & 5 & $(400,400,2801)$ & $\Phi_{5} \cong \mathrm{Gal}\left(\mathbb{F}_{7^{5}} / \mathbb{F}_{7}\right)$ \\
$\mathbb{P}^{6}\left(\mathbb{F}_{2}\right)$ & 63 & 127 & 7 & $(63,63,127)$ & $\Phi_{7} \cong \mathrm{Gal}\left(\mathbb{F}_{2^{7}} / \mathbb{F}_{2}\right)$ \\
$\mathbb{P}^{6}\left(\mathbb{F}_{3}\right)$ & 364 & 1093 & 7 & $(364,364,1093)$ & $\Phi_{7} \cong \mathrm{Gal}\left(\mathbb{F}_{3^{7}} / \mathbb{F}_{3}\right)$ \\
$\mathbb{P}^{6}\left(\mathbb{F}_{5}\right)$ & 3906 & 19531 & 7 & $(3906,3906,19531)$ & $\Phi_{7} \cong \mathrm{Gal}\left(\mathbb{F}_{5^{7}} / \mathbb{F}_{5}\right)$ \\
$\mathbb{P}^{10}\left(\mathbb{F}_{2}\right)$ & 1023 & $2047=23 \cdot 89$ & 11 & $(1023,1023,2047)(?)$ & $\Phi_{11} \cong \operatorname{Gal}\left(\mathbb{F}_{2^{11}} / \mathbb{F}_{2}\right)$ \\
\hline
\end{tabular}

$(?)=$ only if there exists a Frobenius compatible ordering which is also Wada compatible.

Table 2: Some projective spaces whose parameters satisfy the conditions of Proposition 4.4 .

the cyclic group $\Phi_{f}$ or a subgroup $\Phi_{g} \subset \Phi_{f}$ is a group of automorphisms of associated Wada dessins.

Let $\Phi_{g} \subset \Phi_{f}$ be generated by a power $\sigma^{s}$ of the Frobenius automorphism, with $s \in(\mathbb{Z} / f \mathbb{Z})$ and $\operatorname{gcd}(s, f) \neq 1$. The action of $\Phi_{g}$ on the elements of the Frobenius difference set we use to construct the associated dessin is a multiplication by the integer $t=p^{s}$.

As we have seen in Sections 3.1 and 3.2, for $\Phi_{f}$ to be a group of automorphisms of the constructed dessin we need not only a Frobenius difference set $D_{f}$, but we also need the ordering of the elements of $D_{f}$ to be compatible with the action of $\Phi_{f}$. Equivalent conditions are necessary if we need a subgroup $\Phi_{g} \subset \Phi_{f}$ to be a group of automorphisms of the dessin. This means that under the action of $\Phi_{g}$ the elements of a fixed difference set $D_{g}$ have to be subdivided into orbits of equal length. This can be achieved if the following conditions hold:

1. The order $g$ of $\Phi_{g}$ divides $q$, the number of elements of $D_{g}$;

2. The integer $t$ satisfies $g c d(t-1, \ell)=1$.

We need the first condition since it implies that $\Phi_{g}$ has a suitable size to subdivide the elements of $D_{g}$ into orbits of the same length $g$.

The second condition makes sure that all orbits have the same length. In fact, having $\operatorname{gcd}(t-1, \ell)=1$ means that the relation

$$
t \cdot d_{i} \equiv d_{i} \quad \bmod \ell \quad \forall d_{i} \in D_{g}
$$

is satisfied only for $d_{i} \equiv 0 \bmod \ell$. But we exclude this possibility, otherwise $D_{g}$ would contain the only orbit $\{0\}$ of length one and all other orbits would have length $g$. This would be a contradiction to the first condition $g \mid q$, so we have $d_{i} \not \equiv 0 \bmod \ell$ for all $d_{i} \in D_{g}$ and all orbits have length $g$.

Under these conditions, it is possible to order the elements of a difference set fixed by $\Phi_{g}$ in a way compatible with its action, i.e. such that $\Phi_{g}$ acts permuting the elements 
cyclically. If the ordering is also Wada compatible, then $\Phi_{g}$ is a group of automorphisms of the constructed Wada dessin. Similarly to the action of $\Phi_{f}$ (see Section 4) also $\Phi_{g}$ acts on the cells by rotating them around the fixed vertices $w=b=0$ (for a more detailed description see [12]). We conclude with some examples:

Example 5.1. For the projective space $\mathbb{P}^{4}\left(\mathbb{F}_{4}\right)$ we have $q=85$ and $\ell=341$. The group generated by the Frobenius automorphism is the cyclic group $\Phi_{10}$. The order of $\Phi_{10}$ does not divide $q$, so it cannot be a group of automorphisms of any of the dessins associated with $\mathbb{P}^{4}\left(\mathbb{F}_{4}\right)$. Nevertheless, the subgroup $\Phi_{5} \subset \Phi_{10}$ with $5=m+1$ can be. The power $\sigma^{2}$ of the Frobenius automorphism can be chosen as a generator of $\Phi_{5}$. The action of $\Phi_{5}$ on the vertices of associated dessins is expressed by multiplication with $2^{2}$. Since $\operatorname{gcd}\left(2^{2}-1,341\right)=1$, also the second of the two above conditions is satisfied. This means that the elements of the difference set $D_{5}$ fixed by $\Phi_{5}$ are subdivided into $\Phi_{5}$-orbits of equal length: there are 17 orbits of legth five. The elements may, therefore, be ordered in a way compatible with the action of $\Phi_{5}$. If this ordering is such that all differences of consecutive elements $\left(d_{i}-d_{i+1}\right), d_{i}, d_{i+1} \in D_{5}$ are prime to $\ell=341=31 \cdot 11$, i.e. if it is Wada compatible, we may construct an $(85,85,341)$-Wada dessin for which $\Phi_{5}$ is a group of automorphisms acting freely on the 85 cells.

Example 5.2. For $\mathbb{P}^{6}\left(\mathbb{F}_{4}\right)$ we have $q=1365, \ell=5461$. The Frobenius automorphism generates the cyclic group $\Phi_{14}$ with $\operatorname{gcd}(14,1365)=7$. This means that the subgroup $\Phi_{7} \subset \Phi_{14}$ has a suitable size to be a group of automorphisms of possible Wada dessins associated with $\mathbb{P}^{6}\left(\mathbb{F}_{4}\right)$. As in the above example, we may choose the power $\sigma^{2}$ of the Frobenius automorphism as a generator of $\Phi_{7}$. The action of $\Phi_{7}$ on the vertices of associated dessins is expressed by multiplication with powers $2^{2}$. Since $\operatorname{gcd}\left(2^{2}-1,5461\right)=1$, also the second of the two above conditions is satisfied and $\Phi_{7}$ divides the elements of the associated difference set $D_{7}$ into 195 orbits of length 7 . We order the elements of the difference set $D_{7}$ in a way compatible with the action of $\Phi_{7}$. If this ordering is also Wada compatible we may construct a $(1365,1365,5461)$-Wada dessin for which $\Phi_{7}$ is a group of automorphisms acting freely on the cells.

In general, it is not an easy task to construct difference sets with parameters $(v, k, \lambda)$ or, more specifically, with parameters $(\ell, q, \lambda)$ if they are associated with projective spaces $\mathbb{P}^{m}\left(\mathbb{F}_{n}\right)$. The difference sets used in our examples are known difference sets we took from a difference set list in [1]. In [1] as well as in [8] some construction techniques are described. Of course, knowing that a difference set is a Frobenius difference set associated with a projective space $\mathbb{P}^{m}\left(\mathbb{F}_{n}\right)$ and that its elements may be ordered in a Frobenius compatible way may help in the construction (see e.g. the sections about multipliers of difference sets in [1, Section III.c] and in [8, Section 2.5]). In this case the structure of the difference set is, in fact, completely determined by the action of the cyclic group $\Phi_{f}$ or of a subgroup $\Phi_{g}$ of it, as we have seen in Section 3.2 and in the present section. Nevertheless, for projective spaces $\mathbb{P}^{m}\left(\mathbb{F}_{n}\right)$ with arbitrary parameters $m$ and $n$ it is difficult to predict when such orderings occur, as we have remarked above. More research is needed in this direction.

The question about the existence of Wada compatible orderings of elements has been answerd by [14] and more recently by [6] for difference sets associated with projective planes $\mathbb{P}^{2}\left(\mathbb{F}_{n}\right)$. It is still an open and challenging question for projective spaces of higher dimension. 


\section{Acknowledgements}

This is a part of the PhD thesis of the author at the University of Frankfurt. She would like to warmly thank her advisors Jürgen Wolfart and Gareth Jones for introducing her to the beautiful topic of dessins d'enfants and for many useful hints and discussions.

She would also like to thank Benjamin Mühlbauer, Alessandra Sarti and Ayberk Zeytin for carefully reading the first version of this paper. Many thanks also to the anonymous reviewer of this paper for very useful comments.

\section{References}

[1] L. Baumert, Cyclic Difference Sets, Springer - Verlag, Berlin/Heidelberg/New York, 1971.

[2] G.V. Belyı̆, On Galois extensions of a maximal cyclotomic field, Math. USSR Isvestija 14 (1980), 247-256.

[3] A. Beutelspacher and U. Rosenbaum, Projektive Geometrie, Vieweg, Braunschweig/Wiesbaden, 2nd edition, 2004.

[4] Gerard Buskes and Arnoud C. M. van Rooij, Topological Spaces: From Distance to Neighborhood. Springer, New York/Berlin/Heidelberg etc., Reprint, 1997.

[5] P. Dembowski, Finite Geometries, Springer, Berlin/Heidelberg/New York, 2nd edition, 1997.

[6] R. Goertz, Coprime ordering of cyclic planar difference sets, Discrete Math. 309 (2009), $5248-5252$.

[7] J. W. P. Hirschfeld, Projective Geometries over Finite Fields, Clarendon Press, Oxford, 2nd edition, 2005.

[8] D. R. Hughes and F. C. Piper, Design Theory, Cambridge University Press, Cambridge, New York, etc., 1985.

[9] B. Huppert, Endliche Gruppen I, Springer, Berlin/Heidelberg/New York, 1979.

[10] G. Jones, M. Streit and J. Wolfart, Wilson's graph operations on regular dessins and cyclotomic filelds of definition, Proc. London Math. Soc 100 (2010), 510-532.

[11] S.K. Lando and A.K. Zvonkin, Graphs on Surfaces and Their Applications, Springer, Berlin/Heidelberg/New York, 2004.

[12] C. Sarti, Automorphism Groups of Wada Dessins and Wilson Operations, Dissertation, University of Frankfurt, Germany, 2010.

[13] J. Singer, A theorem in finite projective geometry and some applications in number theory, Trans. Amer. Math. Soc., 43 (1938), 377-385.

[14] M. Streit and J. Wolfart, Cyclic projective planes and Wada dessins, Documenta Math. 6, 39-68.

[15] T. R. S. Walsh, Hypermaps versus bipartite maps, J. Comb. Theory Ser. B 18 (1975), 155-163. 